\title{
T cell-inflamed phenotype and increased Foxp3 expression in infiltrating T-cells of mismatch-repair deficient endometrial cancers
}

\author{
Shiho Asaka ${ }^{1}$ Ting-Tai Yen ${ }^{2} \cdot$ Tian-Li Wang ${ }^{1,2,3} \cdot$ le-Ming Shih ${ }^{1,2,3} \cdot$ Stephanie Gaillard ${ }^{1,2}$
}

Received: 23 August 2018 / Revised: 15 October 2018 / Accepted: 15 October 2018 / Published online: 6 November 2018

(c) United States \& Canadian Academy of Pathology 2018

\begin{abstract}
Mismatch repair-deficient endometrial cancers have a high somatic mutation burden, suggesting that patients with these tumors may benefit from immunotherapy. Elucidating the immune suppressive mechanisms of mismatch repair-deficient endometrial cancers is fundamental to developing future immune-based interventions. This study aimed to determine the immune cell populations associated with mismatch repair-deficient endometrial cancers, especially focusing on targetable regulatory pathways of the immune response. A total of 76 endometrial cancer hysterectomy specimens were evaluated for tumor-infiltrating immune cells by immunohistochemistry. Immune specific markers were used to evaluate each specimen for the number of CD8 + cytotoxic T lymphocytes, forkhead-box P3 (FoxP3) + regulatory $\mathrm{T}$ cells, CD68 + tumorassociated macrophages, as well as programmed death-1 (PD-1) + immune cells, and the percentage of programmed death ligand-1 (PD-L1) + immune cells. Mismatch repair-deficient tumors exhibited a significantly higher number of CD8 + cytotoxic T lymphocytes $(p=0.0006)$, FoxP3 + regulatory T cells $(p=0.0003)$, PD- $1+$ immune cells $(p=0.0069)$, and a higher percentage of PD-L1 + immune cells $(p=0.0007)$ occupying the tumor compared to mismatch repair-proficient endometrial cancers. There was no significant difference in CD68 + tumor-associated macrophages infiltration between the two groups. Endometrial cancers with tumor PD-L1 expression also showed significantly increased infiltration of CD8 + cytotoxic T lymphocytes $(p=0.0002)$, FoxP3 + regulatory T cells $(p=0.0003)$, PD- $1+$ immune cells $(p<0.0001)$, and PD-L1 + immune cells $(p<0.0001)$. Endometrial cancers showing mismatch repair-deficiency and PD-L1 expression in tumor cells exhibit a prominent T cell-inflamed phenotype. More importantly, the increased number of FoxP3 + regulatory $\mathrm{T}$ cells in mismatch repair-deficient endometrial cancers suggests that combination therapy by targeting both regulatory $\mathrm{T}$ cells and immune checkpoints may be warranted to improve clinical efficacy.
\end{abstract}

\section{Introduction}

Endometrial cancer is the most common malignant neoplasm in the female genital tract in developed countries, and its incidence is increasing globally [1]. In the United States, an estimated 61,380 new cases and 10,920 deaths were attributable to endometrial cancer in 2017 [2]. While most

Stephanie Gaillard

stephanie.gaillard@jhmi.edu

1 Department of Oncology, Sidney Kimmel Cancer Center, Johns Hopkins School of Medicine, Baltimore, MD, USA

2 Department of Gynecology and Obstetrics, Sidney Kimmel Cancer Center, Johns Hopkins School of Medicine, Baltimore, MD, USA

3 Department of Pathology, Sidney Kimmel Cancer Center, Johns Hopkins School of Medicine, Baltimore, MD, USA patients are diagnosed at an early stage and are successfully treated with hysterectomy with or without radiotherapy or cytotoxic chemotherapy [3], advanced endometrial cancer patients continue to face unfavorable survival outcomes with current treatments, with 5-year survival rates of $\sim 60$ and $\sim 30 \%$ for stage III and IV, respectively [4-6]. In addition, for patients who progress after first-line treatment or have recurrent disease, there is currently no standard second-line therapy [5, 6]. Therefore, novel and effective therapies for patients with advanced or recurrent endometrial cancer are needed.

Cancer immunotherapy is currently drawing intense attention. Monoclonal antibodies blocking two inhibitory pathways, the cytotoxic T lymphocyte antigen-4 (CTLA-4) and programmed cell death-1 (PD-1) pathways, are now routinely used in the clinic for various malignant neoplasms [7-10]. High somatic mutational load, resulting in increased production of tumor-specific neoantigens, is associated with 
innate and therapeutically induced antitumor immune response [11-13]. High mutational load occurs in cancers with high microsatellite instability because of DNA mismatch repair protein deficiency by sporadic or germline (Lynch syndrome) genomic alteration of MLH1, MSH2, MSH6, or PMS2 [14]. Indeed, Le et al [15, 16]. showed a favorable response to immune checkpoint blockade in high microsatellite instability/mismatch repair-deficient cancers of 12 different tumor sites, including endometrial cancers, and improved survival in colorectal cancers. Based on this data, the United States Food and Drug Administration approved the PD-1 antibody, pembrolizumab, for any solid tumor with high microsatellite instability or mismatch repair-deficiency in 2017. High microsatellite instability is a frequent observation in endometrial cancer occurring in $28 \%$ of cases analyzed in The Cancer Genome Atlas project, most commonly occuring in the endometrioid histologic subtype [17].

Multiple regulatory pathways in the tumor microenvironment influence the tumor-specific immune response and are potential pharmacologic targets to activate antitumor immunity. Specifically, CD8 + cytotoxic T lymphocytes and natural killer cells play a central role in recognizing and attacking tumor cells. Interaction between PD-1 expressed on cytotoxic $\mathrm{T}$ lymphocytes and programmed death ligand-1 (PD-L1) and PD-L2 on tumor cells can lead to suppression of the anti-tumor immune response mediated by cytotoxic T lymphocytes [18]. Furthermore, $\mathrm{CD} 4$ + regulatory $\mathrm{T}$ cells, expressing the specific transcription factor forkhead-box P3 (FOXP3), engage in the maintenance of immunological self-tolerance by suppressing self-reactive $\mathrm{T}$ cells, and hamper effective anti-tumor immune response[19, 20]. Several studies have examined the tumor microenvironment in endometrial cancers and revealed clinicopathological significance of tumorinfiltrating lymphocytes and CD68 + tumor-associated macrophages [21-25]. Increased CD3+ or CD8 + tumorinfiltrating lymphocytes in endometrial cancers are associated with favorable prognosis [21, 24], while increased FoxP3 + tumor-infiltrating lymphocytes are associated with worse prognosis [23, 25] and increased CD68 + tumorassociated macrophages are associated with more advanced stage at diagnosis [24].

Only a few studies have examined the relationship between the tumor microenvironment and mismatch repair-status in endometrial cancers [14, 26, 27]. These studies revealed increased tumor-infiltrating lymphocytes, especially increased numbers of tumor-infiltrating cytotoxic $\mathrm{T}$ lymphocytes in mismatch repair-deficient endometrial cancers compared to mismatch repair-proficient endometrial cancers. However, studies comprehensively describing the tumor microenvironment of mismatch repair-deficient endometrial cancers are limited, and markers on tumor-infiltrating lymphocytes regulating anti-tumor immune response in mismatch repairdeficient endometrial cancers have not been well examined. A better understanding of the tumor microenvironment of mismatch repair-deficient endometrial cancers could identify biomarkers predictive of response to immune checkpoint inhibitors and potential candidates for combination therapy with immune checkpoint inhibitors. The present study aimed to compare the tumor microenvironment in mismatch repairproficient and mismatch repair-deficient endometrial cancers focusing on tumor-infiltrating FoxP3 + regulatory $\mathrm{T}$ cells as well as CD8 + cytotoxic T lymphocytes, PD-L1+, PD-1 + immune cells, and CD68 + tumor-associated macrophages.

\section{Materials and methods}

\section{Patient samples and tissue microarray construction}

We used samples from a gynecologic cancer tissue biorepository collected at the Johns Hopkins Medical Institutions under an Institutional Review Board approved protocol. De-identified information was received limited to only relevant clinical descriptors [age, body mass index, obstetric and gynecologic history, other medical history, tumor grades, myometrial invasion, lymphovascular space invasion, and FIGO stages (2009)] and cancer-related longterm follow-up data including disease progression and vital status.

Tissue microarrays were constructed from archived paraffin tissue blocks containing endometrial cancer. Specimens from 76 primary endometrial cancers obtained from hysterectomies performed at the Johns Hopkins Hospital between 1997 and 2003 were randomly selected for this study. Specimens were excluded if the patient had received chemotherapy or radiotherapy before surgery. Pathologists with expertise in gynecologic pathology (S.A. and IM. S.) confirmed the diagnosis of endometrial cancer through review of slides stained with hematoxylin and eosin $(\mathrm{H} \& \mathrm{E})$. Tissue cores ( $1.5 \mathrm{~mm}$ diameter) from three representative tumor-rich regions of each tumor block were obtained from each case to minimize sampling error.

\section{Immunohistochemistry}

Tissue microarrays were stained for four mismatch repair proteins, including MLH-1 (mouse monoclonal, clone M1, catalogue no. 790-4535, predilute; Ventana Medical Systems, Inc.), MSH-2 (mouse monoclonal, clone G219-1129, catalogue no. 760-4265, predilute; Ventana Medical Systems, Inc.), MSH-6 (mouse monoclonal, clone 44, catalogue no. 7904455, predilute; Ventana Medical Systems, Inc.), and PMS-2 (rabbit monoclonal, clone EPR3947, catalogue no. 760-4531, predilute; Ventana Medical Systems, Inc.). Tumor-infiltrating 


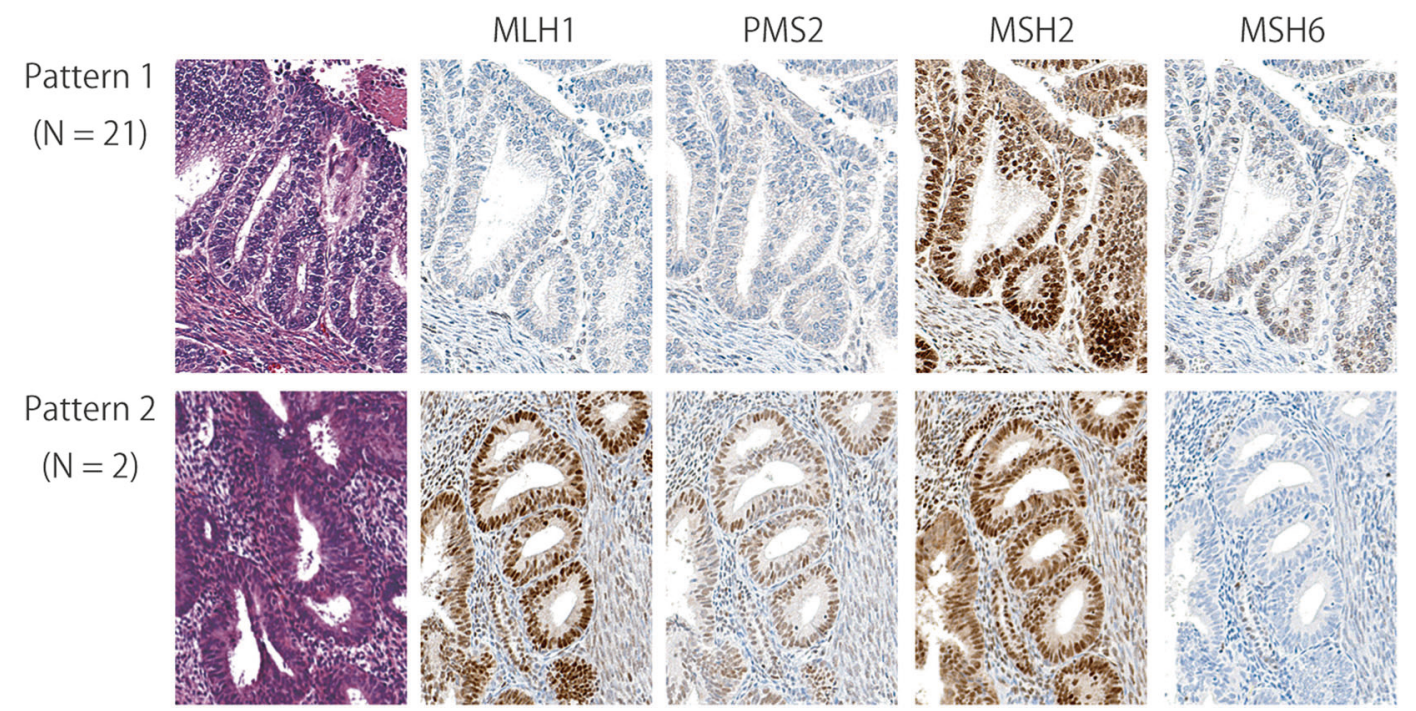

Fig. 1 Immunohistochemical patterns of mismatch repair deficient endometrial cancers. Hematoxylin and eosin staining and immunohistochemistry of MLH1, PMS2, MSH2, and MSH6. Pattern 1. A representative mismatch repair deficient case with the loss of MLH1 and PMS2. Twenty-one endometrial cancers showed pattern 1 mismatch repair-deficiency. While MLH1 and PMS2 are expressed in nuclei of stromal cells, tumor cells are negative for those markers.

immune cells were examined using these antibodies: CD8 (mouse monoclonal, clone C8/144B, catalogue no. 108M-98, predilute; Cell Marque), FoxP3 (mouse monoclonal, clone 236 A/E7, catalogue no. 14-4777-80, 1:100; abcam, Cambridge, MA, USA), CD68 (mouse monoclonal, clone KP-1, catalogue no. 790-2931, predilute; Ventana Medical Systems, Inc.), PD-1(mouse monoclonal, clone NAT105, catalogue no. 315M-96, 1:100; Cell Marque, Rocklin, CA, USA), and PDL1 (rabbit monoclonal, clone SP263, catalogue no. 790-4905, predilute; Ventana Medical Systems, Inc., Tucson, AZ, USA). The antibodies against the mismatch repair proteins including MLH-1, MSH-2, MSH-6, and PMS-2, CD8, CD68, and PD-1 have been validated and the panel of antibodies are used as a routine clinical test at the Johns Hopkins Hospital.

Hormone receptor expression is commonly requested in practice to predict response to endocrine therapy. We followed the College of American Pathologists template for reporting results of biomarker testing of specimens from patients with carcinoma of the endometrium (v1.1.0.0 June 2016) and performed immunostaining of estrogen receptor alpha (phospho S118) (rabbit polyclonal, catalogue no. ab31477, 1:1000; abcam) and progesterone receptor (rabbit polyclonal, catalogue no. ab31477, 1:800; abcam) in the tissue microarrays. Since we have previously reported that ARID1A loss correlates with mismatch repair deficiency in endometrial cancers [28], we also performed immunohistochemistry for ARID1A (rabbit polyclonal, catalogue no. HPA005456, 1:2000; Sigma-Aldrich Co. LLC, St. Louis, MO, USA) to validate the settings of the present study.
MSH2 and MSH6 are expressed in nuclei of tumor and stromal cells. Pattern 2. A representative mismatch repair deficient case with the loss of MSH6. Two endometrial cancers showed pattern 2 mismatch repairdeficiency. While MSH6 are expressed in stromal cells and normal endometrial glands, tumor cells are negative for MSH6. MLH1, MSH2, and PMS2 were expressed in nuclei of tumor and stromal cells

We made serial paraffin sections of $4 \mu \mathrm{m}$ thickness from each tissue microarray, and sections were stained with $\mathrm{H} \& \mathrm{E}$ for histological examination and subjected to immunohistochemistry. Immunohistochemistry was performed using an automated instrument (BenchMark Ultra; Ventana Medical Systems, Inc.) according to manufacturer's instruction for MLH-1, MSH-2, MSH-6, PMS-2, CD8, FoxP3, CD68, PD-1, and PD-L1. Immunohistochemistry of estrogen receptor, progesterone receptor, and ARID1A was performed manually as follows: briefly, unstained sections were deparaffinized and subject to antigen retrieval by incubating slides in DAKO Target Retrieval Solution, Citrate pH 6 (Agilent, Santa Clara, CA, USA) for estrogen receptor and progesterone receptor and in Trilogy ${ }^{\mathrm{TM}}$ (Sigma-Aldrich, Darmstadt, Germany) for ARID1A at $90^{\circ} \mathrm{C}$ for $30 \mathrm{~min}$. Tissue sections were then incubated with the primary antibody at $4{ }^{\circ} \mathrm{C}$ overnight. Immunoreactivity was detected by using DAKO EnVision ${ }^{\mathrm{TM}}$ + System- HRP Labelled Polymer Anti-Rabbit (Agilent, Santa Clara, CA, USA).

\section{Scoring}

Mismatch repair-deficiency was defined as complete absence of immunoreactivity of at least one of the four mismatch repair proteins (MLH-1, MSH-2, MLH-6, PMS-2) in tumor cells. The immunoreactivity of stromal cells was used as positive controls. Total numbers of $\mathrm{CD} 8+$, FoxP3+, CD68+, and PD-1+ tumor-infiltrating immune cells were quantified per core, and the mean of the three cores were analyzed for 
Table 1 Clinicopathological characteristics

\begin{tabular}{llll}
\hline Total & $\begin{array}{l}\text { MMR } \\
\text { proficient }\end{array}$ & $\begin{array}{l}\text { MMR } \\
\text { deficient }\end{array}$ & $p$ value \\
& $\begin{array}{l}N=76 \\
(\%)\end{array}$ & $N=53(\%)$ & $N=23(\%)$
\end{tabular}

Age

$\begin{array}{lllll}<65 \text { years } & 42(55) & 29(55) & 13(57) & 1.0000^{\mathrm{a}} \\ \geq 65 \text { years } & 34(45) & 24(45) & 10(44) & \end{array}$

Race

White

Black

(Other)

Parity

Nulliparous

Multiparous

(Unknown)

Menstrual state

Pre-menopausal

Post-menopausal

59 (78) 40 (76)

19 (83)

$0.7623^{\mathrm{a}}$

6 (21) $12(23)$

4 (17)

1 (1) 1 (2)

$0(0)$

7 (30)

17 (22) $10(19)$

58 (78) $42(81)$

$16(70)$

$0(0)$

2 (9)

21 (91)

Body mass index

$<25 \mathrm{~kg} / \mathrm{m}^{2}$
$\geq 25 \mathrm{~kg} / \mathrm{m}^{2}$
(Unknown)

Smoking history

Never smoker

Current or former smoker

(Unknown

Endometrioid
Non-endometrioid

$11(15) \quad 9$ (17)

65 (86) 44 (83)

15 (20) $11(21)$

4 (17)

17 (74)

48 (63) 31 (56)

2 (9)

13 (17) $11(21)$

20 (87)

$0.3610^{\mathrm{a}}$

3 (13)

$0(0)$

Histology grade

Low (G1, G2)

High (G3)

58 (76) 38 (72)

15 (20) $12(23)$

$3(4) \quad 3(6)$

60 (79) $38(72)$

22 (96)

1 (4)

$14(61)$

9 (39)

Myometrial invasion

Absent

23 (30) 17 (32)

6 (26)

$0.7867^{\mathrm{a}}$

Present

$53(70) \quad 36(67)$

$17(74)$

Lymphovascular space invasion

Absent or

60 (79) $45(85)$

$15(65)$

$0.0692^{\mathrm{a}}$

unknown

Present

$16(21) \quad 8(15)$

$8(35)$

FIGO 2009 stage

$\begin{array}{lllll}\text { I } & 54(71) & 36(68) & 18(78) & 0.4967^{\mathrm{b}} \\ \text { II } & 5(7) & 5(9) & 0(0) & \\ \text { III } & 11(15) & 8(15) & 3(13) & \\ \text { IV } & 6(8) & 4(8) & 2(9) & \\ \text { Estrogen receptor } & & & & \\ \text { Negative } & 1(1) & 1(2) & 0(0) & 1.0000^{\mathrm{a}} \\ \text { Positive } & 75(99) & 52(98) & 23(100) & \end{array}$

Progesterone receptor
Table 1 (continued)

\begin{tabular}{lllll}
\hline Negative & $7(9)$ & $5(9)$ & $2(9)$ & $1.0000^{\mathrm{a}}$ \\
$\begin{array}{l}\text { Positive } \\
\text { ARID1A }\end{array}$ & $69(91)$ & $48(90)$ & $21(91)$ & \\
$\quad$ & & & & \\
Retain & $50(66)$ & $42(79)$ & $8(35)$ & $0.0004^{\mathrm{a}}$ \\
Loss & $26(34)$ & $11(21)$ & $15(65)$ & \\
$\begin{array}{l}\text { Tumor PD-L1, case no. } \\
\quad\end{array}$ & & & \\
$\quad$ Negative & $53(70)$ & $42(79)$ & $14(61)$ & $0.1546^{\mathrm{a}}$ \\
Positive & $23(30)$ & $11(21)$ & $9(39)$ & \\
\hline
\end{tabular}

${ }^{\text {a }}$ Fisher's exact test

${ }^{\mathrm{b}}$ Wilcoxon test

individual cases. PD-L1 was evaluated on tumor cells and tumor-infiltrating immune cells respectively as previously described [29]. In the present study, the percentage of membranous PD-L1 positive tumor cells in three cores was recorded in each case, and tumors with at least $1 \%$ of PD-L1 expression were considered as positive for tumor PD-L1. For PD-L1 + tumor-infiltrating immune cells, which showed typically membranous positivity of PD-L1 rather than cytoplasmic granular positivity, the percentage of PD-L1 + immune cells occupying the tumor in three cores was recorded in each case. Nuclear estrogen receptor and progesterone receptor expression in tumor cells were evaluated as either positive or negative. In the present study, focal or weak expression was considered positive. Tumor ARID1A was evaluated as either retained or lost. Nuclear ARID1A immunoreactivity was considered positive and loss of ARID1A expression was defined as the complete or clonal absence of ARID1A immunoreactivity in virtually all tumor cells examined.

\section{Statistical analysis}

Fisher's exact tests and Wilcoxon tests (Mann-Whitney test) were performed to compare clinicopathological characteristics between mismatch repair-intact and deficient groups. Wilcoxon tests was also performed to compare the distribution of tumor-infiltrating immune cells between the two groups. The $p$-value $<0.05$ was considered statistically significant. The data were statistically analyzed using JMP software (version 13.0; SAS Institute, Cary, NC, USA).

\section{Results}

\section{Clinicopathological characteristics and mismatch repair protein status}

The tissue microarray comprised the following endometrial cancer histologic subtypes: 60 endometrioid 
carcinoma, 7 serous carcinoma, 4 carcinosarcoma, 2 clear cell carcinoma, 2 mixed cell carcinoma, and 1 undifferentiated carcinoma. Twenty-three samples showed loss of expression of at least one mismatch repair protein: 21 with MLH1 and PMS2 deficiency, and two with MSH6 deficiency. Figure 1 shows immunostaining patterns of representative cases of mismatch repair-deficient endometrial cancer.

The clinicopathological characteristics and correlation with mismatch repair status are summarized in Table 1 . The mean age of the 76 patients was 63.6 years (range, 42-89 years), the majority of patients were Caucasian $(78 \%)$, multiparous $(78 \%)$, and post-menopausal $(86 \%)$. There were no significant differences between samples with mismatch repair-proficient and -deficient endometrial cancer in age, race, parity, menstrual status, body mass index, smoking history, histology grade, myometrial invasion, lymphovascular space invasion, FIGO stage, estrogen receptor/progesterone receptor expression, and tumor PDL1 expression. Endometrioid histology and loss of ARID1A expression were significantly increased in samples with mismatch repair-deficiency.

\section{The relationship between mismatch repair status and tumor-infiltrating immune cells}

Figure 2 shows representative staining of tumor-infiltrating immune cells in mismatch repair-proficient and deficient endometrial cancers (A) and the relationship between mismatch repair status and tumor-infiltrating immune cells (B). Infiltrating immune cells were seen in both the peritumoral stroma and the intratumoral compartment. In the intratumoral compartments, immune cells infiltrated in the gap between tumor cells without intervening stroma. More PD-L1 + immune cells were seen in the peritumoral stroma compared to the intratumoral compartment. Mismatch repair-deficient endometrial cancers exhibited significantly higher numbers of CD8 + cytotoxic $\mathrm{T}$ lymphocytes, FoxP3 + regulatory $\mathrm{T}$ cells, PD- $1+$ immune cells, and an increased percentage of PD-L1 + immune cells occupying the tumor compared to mismatch repair-proficient endometrial cancers. The median value of the average positive-staining cells per core in mismatch repair-deficient vs proficient tumors were 156.6 vs. 73.3 for CD8 + cytotoxic T lymphocytes $(p=0.0006), 47.5$ vs. 17.3 for FoxP3 regulatory $\mathrm{T}$ cells $(p=0.0003), 53.3$ vs. 3.0 for PD- $1+$ immune cells $(p=0.0069)$, and 5 vs. $1 \%$ of tumor cells for PD-L1 + immune cells $(p=0.0007)$, respectively. There was no significant difference in the number of CD68 + tumor-associated macrophages between mismatch repair proficient and deficient endometrial cancers $(p=0.5046)$.

\section{The relationship between tumor PD-L1 expression and tumor-infiltrating immune cells}

Figure 3 shows representative staining of tumor-infiltrating immune cells in endometrial cancer with or without tumor PD-L1 expression (A) and the relationship between tumor PD-L1 expression status and tumor-infiltrating immune cells (B). Endometrial cancers with tumor PD-L1 expression showed significantly higher numbers of CD8 + cytotoxic T lymphocytes, FoxP3 + regulatory T cells, PD-1 + immune cells, and a higher percentage of PD-L1 + immune cells occupying the tumor compared to endometrial cancers without tumor cell PD-L1 expression. The median value of the average positive-staining cells per core in endometrial cancers with and without PD-L1 expression on tumor cells was 195.0 vs. 71.7 for CD8 + cytotoxic T lymphocytes $(p=0.0002), \quad 54.2$ vs. 17.4 for FoxP3 + regulatory $\mathrm{T}$ cells $(p=0.0003), 79.3$ vs. $2.8 \mathrm{PD}-1+$ immune cells $(p<0.0001)$, and $10 \%$ vs. $0 \%$ PD-L1 + immune cells $(p<0.0001)$, respectively. There was no significant difference in CD68 + tumor-associated macrophages between endometrial cancers with and without tumor cell PD-L1 expression $(p=0.1693)$.

\section{Discussion}

In the present study, the tumor microenvironment of mismatch repair-deficient endometrial cancers were compared to mismatch repair-proficient endometrial cancers. We found that mismatch repair-deficiency was significantly associated with loss of ARID1A immunoreactivity (presumably due to inactivating mutations) while it was not associated with either estrogen receptor or progesterone receptor expression. These results are consistent with several reports [28, 30,31], indicating the robustness of this cohort and our staining procedures. The results show significantly increased infiltration of CD8 + cytotoxic $\mathrm{T}$ lymphocytes, FoxP3 + regulatory T cells, PD-1+, and PD$\mathrm{L} 1+$ immune cells in mismatch repair-deficient endometrial cancers. Similarly, endometrial cancers with tumor PD-L1 expression exhibited higher numbers of CD8 + cytotoxic T lymphocytes, FoxP3 + regulatory T cells, PD$1+$, and PD-L1 + immune cell infiltration compared to endometrial cancers without tumor PD-L1 expression. Importantly, there was no significant correlation between mismatch repair status and tumor PD-L1 expression in the present study suggesting that these are independent markers of an inflamed phenotype.

The results showing increased CD8 + cytotoxic T lymphocytes, PD-1+, and PD-L1 + tumor-infiltrating immune cells in mismatch repair-deficient endometrial cancers are 


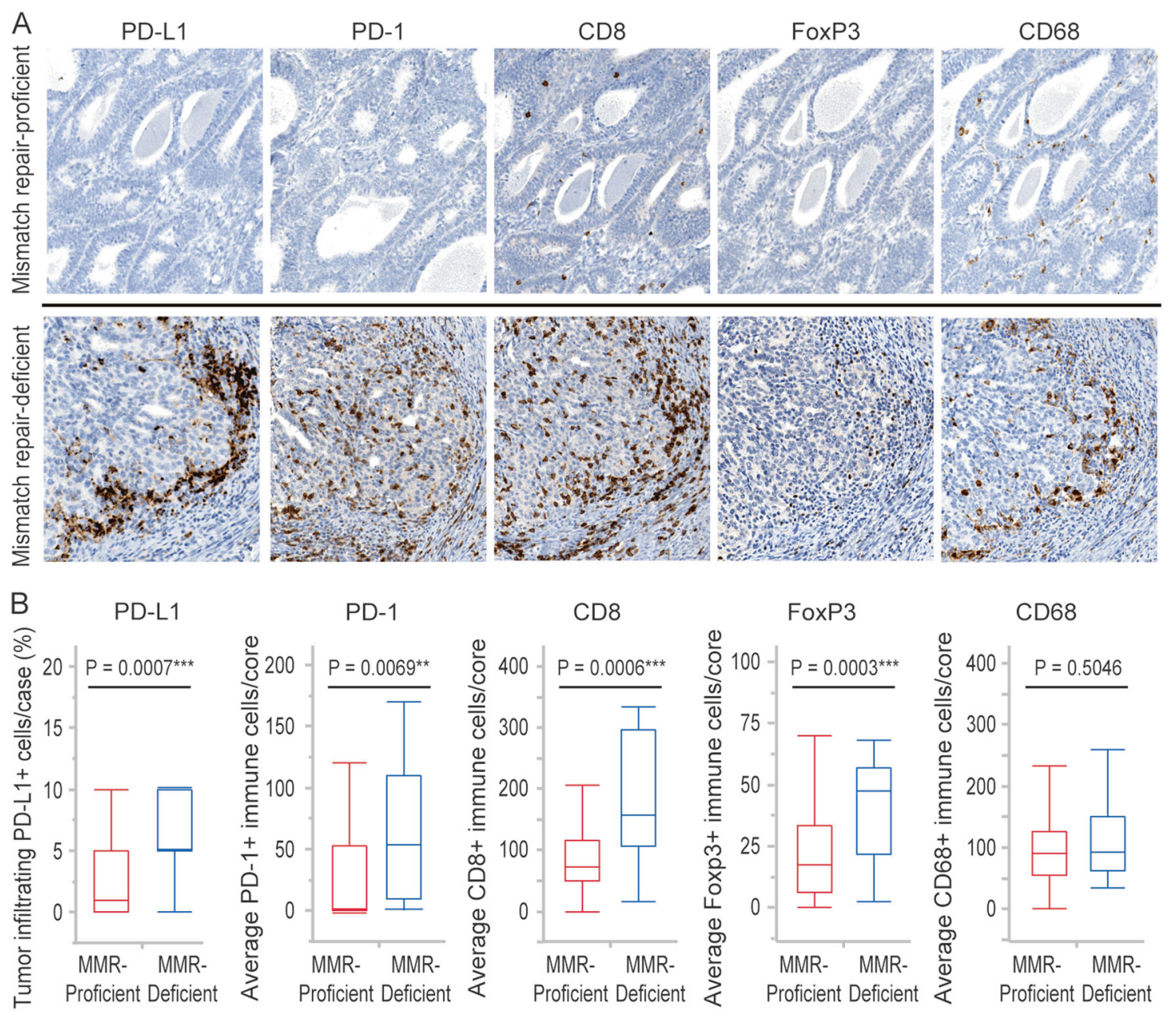

Fig. 2 Infiltration of PD-L1+, PD-1+, CD8+, FoxP3+, and CD68+ immune cells in mismatch repair proficient and deficient endometrial cancers. a Immunostaining of PD-L1+, PD-1+, CD $8+$, FoxP3+, and CD68 + immune cells in representative cases of mismatch repairproficient and mismatch repair-deficient endometrial cancers. b Percent of tumor-infiltrating PD-L1 + immune cells occupying the tumor in three cores and average numbers of PD1+, CD8+, FoxP3+, and CD68 cells per core in each case. Boxes represent the interquartile range, with the upper whisker indicating the 75 th percentile and the lower whisker indicating the 25 th percentile. The median values are indicated by a horizontal line. MMR, mismatch repair; * $p$-value $<$ $0.05 ; * * p$-value $<0.01 ; * * *$-value $<0.001$ consistent with previous studies. Several studies reported enhanced intratumoral infiltration of CD8+, PD-1+, and PD-L1 + immune cells in patients with high microsatellite instability [14, 26, 27, 32] and POLE-ultramutated [14, 26] endometrial cancer. Enriched CD8+ and PD-1 + cell infiltration in mismatch repair-deficient endometrial cancers suggest enriched infiltration of tumor-specific cytotoxic $\mathrm{T}$ lymphocytes targeting neoantigens in those tumors. Indeed, the association between higher neoantigen load and immune cell infiltration is supported by a recent large-scale genomic characterization of colorectal cancers, in which Giannakis et al. [33] revealed high neoantigen load correlates with increased lymphocytic infiltration and improved survival.

Increased infiltration of FoxP3 + regulatory $\mathrm{T}$ cells has been reported to be associated with poor histology grade, frequent lymphovascular space invasion, advanced FIGO stage, and worse prognosis in endometrial cancer [21, 23]. However, the presence of increased FoxP3 + regulatory $\mathrm{T}$ cells in mismatch repair-deficient compared to -proficient endometrial cancer in our study is a novel finding. This is supported by other studies showing an association between the T-cell inflamed tumor microenvironment and increased FoxP3 + regulatory $\mathrm{T}$ cells along with other negative regulatory factors due to recruitment of regulatory $\mathrm{T}$ cells as well as cytotoxic $\mathrm{T}$ lymphocytes. It has been proposed that the chemokine CCL22 produced by tumor cells and tumorassociated macrophages recruits regulatory $\mathrm{T}$ cells $[34,35]$ and that high concentrations of TGF- $\beta$ secreted by tumor and/or dendritic cells induce differentiation from naïve $\mathrm{T}$ cells to regulatory $\mathrm{T}$ cells $[36,37]$. Whether survival is 


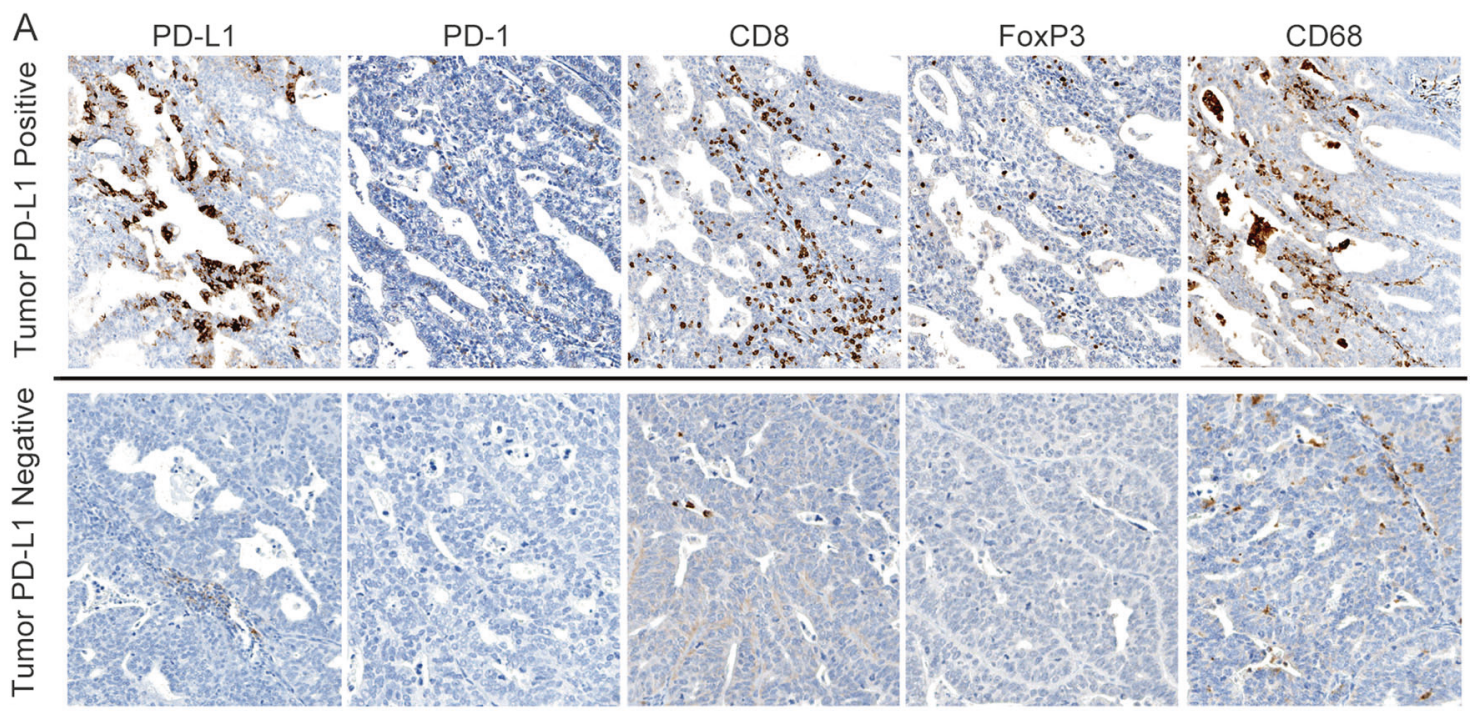

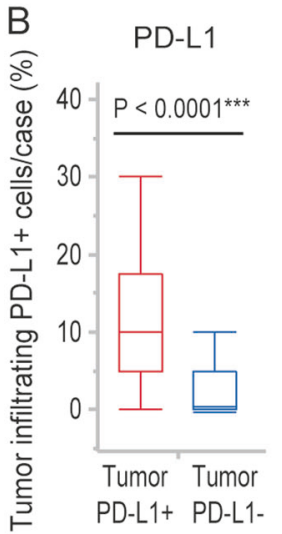
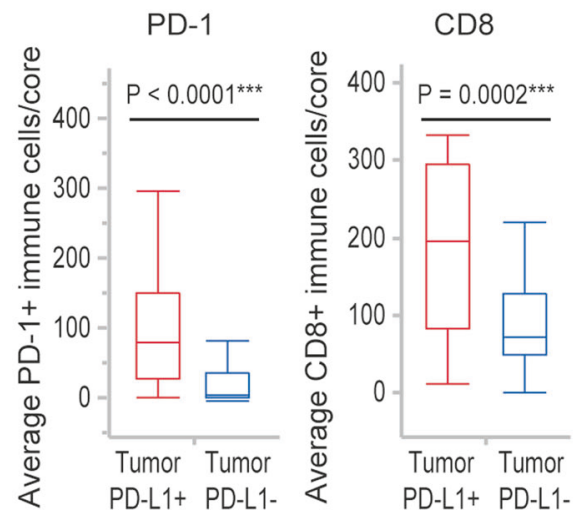
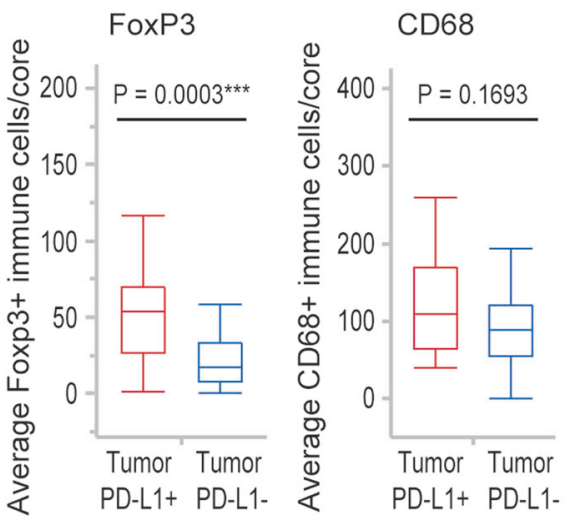

Fig. 3 Infiltration of PD-L1+, PD-1+, CD8+, FoxP3+, and CD68 + immune cells in tumor PD-L1 positive and negative endometrial cancers. a Immunostaining of PD-L1+, PD-1+, CD8+, FoxP3+, and CD68 + immune cells in representative cases of tumor PD-L1 positive and negative endometrial cancers. b Percent of tumor-infiltrating PD$\mathrm{L} 1+$ immune cells occupying the tumor in three cores and average numbers of PD1+, CD8+, FoxP3+, and CD68 cells per core in each case. Boxes represent the interquartile range, with the upper whisker indicating the 75th percentile and the lower whisker indicating the 25 th percentile. The median values are indicated by a horizontal line. ${ }^{*} p$-value $<0.05 ; * * p$-value $<0.01 ; * * * p$-value $<0.001$ affected by FoxP3 expression could not be determined in the present study and awaits a future clinical study using a larger mismatch repair-deficient tumor cohort.

In the present study, endometrial cancers with tumor PDL1 expression also showed increased infiltration of stromal CD8 + cytotoxic T lymphocytes, FoxP3 + regulatory T cells, PD-1+, and PD-L1 + immune cells compared to tumors without PD-L1 expression. The lack of association between mismatch repair status and tumor PD-L1 expression suggests that PD-L1 + tumors can have a T cell inflamed phenotype, regardless of mismatch repair status. Two studies have examined the prognostic effect of endometrial cancer with PD-L1 expression in tumor cells. However, there has been no significant difference in overall survivals between PD-L1 positive and negative endometrial cancers [27, 38], although progression-free survival was significantly prolonged in patients with PD-L1 positive endometrial cancer [27]. In mouse studies of melanoma, the presence of CD8 $+\mathrm{T}$ cells in tumor microenvironment induced the up-regulation of PD-L1 and indoleamine-2,3-dioxygenase (IDO) in tumor cells and increased recruitment of regulatory $\mathrm{T}$ cells to the tumor microenvironment [39]. The regulation of PD-L1 and IDO were driven by IFN- $\gamma$, while the recruitment of regulatory $\mathrm{T}$ cells was driven by the chemokine CCL22 produced by $\mathrm{CD} 8+\mathrm{T}$ cells. The authors proposed that immunosuppressive pathways are intrinsically driven by activated CD8 + $\mathrm{T}$ cells rather than by the tumor cells and that targeting negative regulatory immune checkpoints might be beneficial for patients with a preexisting $\mathrm{T}$ cell-inflamed tumor microenvironment. Thus, as seen in our results, there is an association between the $\mathrm{T}$ cell-inflamed tumor microenvironment and PD-L1 positivity in endometrial cancers, however based 
on their results, PD-L1 expression in tumor cells might be not the cause but rather the effect of an inflamed tumor microenvironment.

The $\mathrm{T}$ cell-inflamed phenotype and increased infiltration of Foxp3 + regulatory $\mathrm{T}$ cells in both endometrial cancers with mismatch repair -deficient and PD-L1 expressing tumors suggests combination therapies to target multiple inhibitory mechanisms may improve anti-tumor immune response. In particular, monoclonal antibodies against CCR4 and CTLA-4, both of which are specifically expressed by activated FoxP3 + regulatory $\mathrm{T}$ cells, may result in regulatory $\mathrm{T}$ cell depletion [20]. Indeed, the combination of anti-PD-1/PD-L1 and anti-CTLA-4 blockade has been beneficial for multiple cancer types [40-43], though whether this is due specifically to regulatory $\mathrm{T}$ cell depletion effects is unknown. In the present study, none of the patients received immunotherapy to determine whether FoxP3 expression influenced response to therapy. Further studies examining the relationship between clinical response to immune checkpoint blockade and FoxP3 + regulatory $\mathrm{T}$ cell infiltration in individual endometrial cancers are necessary.

A potential limitation of this study is the employment of tissue microarrays for evaluating tumor microenvironment and mismatch repair proteins in endometrial cancers as small cores might not reflect the tumor microenvironment and mismatch repair status of whole endometrial cancer tumor tissue. However, studies showed immunologic homogeneity in multiple tumor sites using tissue microarray in serous ovarian carcinoma [44], and prostate cancer [45]. Also, previous studies showing significant correlation between tumor-infiltrating lymphocytes and clinical outcome used tissue microarrays for immunohistochemistry evaluation [46-48]. The validity of tissue microarrays to assess mismatch repair status has been evaluated in endometrial carcinoma [49] as well as colorectal carcinoma [50], Visser et al. [51] have recently found almost perfect agreement in mismatch repair protein assessment by immunohistochemistry between whole slides versus tissue microarrays using either $2.0 \mathrm{~mm}$ or $0.6 \mathrm{~mm}$ duplicate cores from each hysterectomy specimen. To minimize the effect of heterogeneity in tumor microenvironment in our study, 1.5 cores from three separate sites per case were included in the tissue microarray and the mean number of tumorinfiltrating immune cells for each case was calculated.

In conclusion, among endometrial cancers, increased FoxP3 + regulatory $\mathrm{T}$ cell infiltration as well as $\mathrm{CD} 8+$ cytotoxic T lymphocytes, PD-1+, and PD-L1 + immune cells were observed in mismatch repair-deficient endometrial cancer and PD-L1 expressing endometrial cancer. These results are consistent with an inflamed phenotype. More efforts are needed to illuminate the interaction and trafficking among these immune cell populations and to better characterize the biological significance of our findings in mouse endometrial cancer models. Further evaluation to determine whether FoxP3 + regulatory $\mathrm{T}$ cell infiltration influences response to immunotherapy and if so, combination therapy targeting Tregs with immune checkpoint inhibitors could improve clinical outcomes.

Acknowledgements The authors acknowledge Hiro Nonogaki and Rajni Sharma (Department of Pathology, Johns Hopkins School of Medicine, Baltimore, MD, USA) for technical assistance in performing some of the immunohistochemical stains.

\section{Compliance with ethical standards}

Conflict of interest The authors declare that they have no conflict of interest.

\section{References}

1. Torre LA, Bray F, Siegel RL, et al. Global cancer statistics, 2012. CA. 2015;65:87-108.

2. Siegel RL, Miller KD, Jemal A. Cancer statistics, 2017. CA. 2017;67:7-30.

3. Wright JD, Barrena Medel NI, Sehouli J, et al. Contemporary management of endometrial cancer. Lancet. 2012;379:1352-60.

4. Galaal K, Al Moundhri M, Bryant A, et al. Lopes AD, Lawrie TA. Adjuvant chemotherapy for advanced endometrial cancer. Cochrane Database Syst Rev. 2014;5:Cd010681.

5. Dowdy SC. Improving oncologic outcomes for women with endometrial cancer: realigning our sights. Gynecol Oncol. 2014;133:370-4.

6. Bradford LS, Rauh-Hain JA, Schorge J, et al. Advances in the management of recurrent endometrial cancer. Am J Clin Oncol. 2015;38:206-12.

7. Lipson EJ, Drake CG. Ipilimumab: an anti-CTLA-4 antibody for metastatic melanoma. Clin Cancer Res. 2011;17:6958-62.

8. Ott PA, Hodi FS, Robert C. CTLA-4 and PD-1/PD-L1 blockade: new immunotherapeutic modalities with durable clinical benefit in melanoma patients. Clin Cancer Res. 2013;19:5300-9.

9. Topalian SL, Sznol M, McDermott DF, et al. Survival, durable tumor remission, and long-term safety in patients with advanced melanoma receiving nivolumab. J Clin Oncol. 2014;32: 1020-30.

10. Balar AV, Weber JS. PD-1 and PD-L1 antibodies in cancer: current status and future directions. Cancer Immunol Immunother. 2017;66:551-64.

11. Schumacher TN, Schreiber RD. Neoantigens in cancer immunotherapy. Science. 2015;348:69-74.

12. Ward JP, Gubin MM, Schreiber RD. The Role of neoantigens in naturally occurring and therapeutically induced immune responses to cancer. Adv Immunol. 2016;130:25-74.

13. Gubin MM, Zhang X, Schuster H, et al. Checkpoint blockade cancer immunotherapy targets tumour-specific mutant antigens. Nature. 2014;515:577-81.

14. Howitt BE, Shukla SA, Sholl LM, et al. Association of polymerase e-mutated and microsatellite-instable endometrial cancers with neoantigen load, number of tumor-infiltrating lymphocytes, and expression of PD-1 and PD-L1. JAMA Oncol. 2015;1:1319-23.

15. Le DT, Uram JN, Wang H, et al. PD-1 Blockade in tumors with mismatch-repair deficiency. N Engl J Med. 2015;372: 2509-20. 
16. Le DT, Durham JN, Smith KN, et al. Mismatch repair deficiency predicts response of solid tumors to PD-1 blockade. Science. 2017;357:409-13.

17. Kandoth C, Schultz N, Cherniack AD, et al. Integrated genomic characterization of endometrial carcinoma. Nature. 2013;497:67-73.

18. Ohaegbulam KC, Assal A, Lazar-Molnar E, et al. Human cancer immunotherapy with antibodies to the PD-1 and PD-L1 pathway. Trends Mol Med. 2015;21:24-33.

19. Wang HY, Lee DA, Peng G, et al. Tumor-specific human CD4 + regulatory $\mathrm{T}$ cells and their ligands: implications for immunotherapy. Immunity. 2004;20:107-18.

20. Nishikawa H, Sakaguchi S. Regulatory $\mathrm{T}$ cells in cancer immunotherapy. Curr Opin Immunol. 2014;27:1-7.

21. de Jong RA, Leffers N, Boezen HM, et al. Presence of tumor-infiltrating lymphocytes is an independent prognostic factor in type I and II endometrial cancer. Gynecol Oncol. 2009;114:105-10.

22. Iurchenko NP, Glushchenko NM, Buchynska LG. Comprehensive analysis of intratumoral lymphocytes and FOXP3 expression in tumor cells of endometrial cancer. Exp Oncol. 2014;36:262-6.

23. Yamagami W, Susumu N, Tanaka H, et al. Immunofluorescencedetected infiltration of CD4+FOXP3+regulatory T cells is relevant to the prognosis of patients with endometrial cancer. Int $\mathbf{J}$ Gynecol Cancer. 2011;21:1628-34.

24. Cermakova P, Melichar B, Tomsova M, et al. Prognostic significance of $\mathrm{CD} 3$ +tumor-infiltrating lymphocytes in patients with endometrial carcinoma. Anticancer Res. 2014;34:5555-61.

25. Giatromanolaki A, Bates GJ, Koukourakis MI, et al. The presence of tumor-infiltrating FOXP3+lymphocytes correlates with intratumoral angiogenesis in endometrial cancer. Gynecol Oncol. 2008;110:216-21.

26. Eggink FA, Van Gool IC, Leary A, et al. Immunological profiling of molecularly classified high-risk endometrial cancers identifies POLE-mutant and microsatellite unstable carcinomas as candidates for checkpoint inhibition. Oncoimmunology. 2017;6: e1264565.

27. Yamashita H, Nakayama K, Ishikawa M, et al. Microsatellite instability is a biomarker for immune checkpoint inhibitors in endometrial cancer. Oncotarget. 2018;9:5652-64.

28. Allo G, Bernardini MQ, Wu RC, et al. ARID1A loss correlates with mismatch repair deficiency and intact p53 expression in high-grade endometrial carcinomas. Mod Pathol. 2014;27:255-61.

29. Herbst RS, Soria JC, Kowanetz M, et al. Predictive correlates of response to the anti-PD-L1 antibody MPDL3280A in cancer patients. Nature. 2014;515:563-7.

30. Huang HN, Lin MC, Tseng LH, et al. Ovarian and endometrial endometrioid adenocarcinomas have distinct profiles of microsatellite instability, PTEN expression, and ARID1A expression. Histopathology. 2015;66:517-28.

31. Okoye EI, Bruegl AS, Fellman B, et al. Defective DNA mismatch repair influences expression of endometrial carcinoma biomarkers. Int J Gynecol Pathol. 2016;35:8-15.

32. Pakish JB, Zhang Q, Chen Z, et al. Immune Microenvironment in microsatellite-instable endometrial cancers: hereditary or sporadic origin matters. Clin Cancer Res. 2017;23:4473-81.

33. Giannakis M, Mu XJ, Shukla SA, et al. Genomic correlates of immune-cell infiltrates in colorectal carcinoma. Cell Rep. 2016;15:857-65.

34. Yang $\mathrm{P}$, Li QJ, Feng $\mathrm{Y}$, et al. TGF-beta-miR-34a-CCL22 signaling-induced Treg cell recruitment promotes venous metastases of HBV-positive hepatocellular carcinoma. Cancer Cell. 2012;22:291-303.

35. Faget J, Biota C, Bachelot T, et al. Early detection of tumor cells by innate immune cells leads to $\mathrm{T}(\mathrm{reg})$ recruitment through CCL22 production by tumor cells. Cancer Res. 2011;71:6143-52.

36. Nishikawa H, Sakaguchi S. Regulatory T cells in tumor immunity. Int J Cancer. 2010;127:759-67.

37. Liu VC, Wong LY, Jang T, et al. Tumor evasion of the immune system by converting CD $4+\mathrm{CD} 25 \mathrm{~T}$ cells into CD $4+\mathrm{CD} 25+\mathrm{T}$ regulatory cells: role of tumor-derived TGF-beta. J Immunol. 2007;178:2883-92.

38. Li Z, Joehlin-Price AS, Rhoades J, et al. Programmed death ligand 1 expression among 700 consecutive endometrial cancers: strong association with mismatch repair protein deficiency. Int J Gynecol Cancer. 2018;28:59-68.

39. Spranger S, Spaapen RM, Zha Y, et al. Up-regulation of PD-L1, IDO, and T(regs) in the melanoma tumor microenvironment is driven by CD8(+) T cells. Sci Transl Med. 2013;5:200ra116.

40. Wolchok JD, Chiarion-Sileni V, Gonzalez R, et al. Overall survival with combined Nivolumab and Ipilimumab in advanced melanoma. N Engl J Med. 2017;377:1345-56.

41. Hellmann MD, Callahan MK, Awad MM, et al. Tumor mutational burden and efficacy of Nivolumab monotherapy and in combination with Ipilimumab in small-cell lung cancer. Cancer Cell. 2018;33:853-61. e4

42. Motzer RJ, Tannir NM, McDermott DF, et al. Nivolumab plus Ipilimumab versus Sunitinib in advanced renal-cell carcinoma. N Engl J Med. 2018;378:1277-90.

43. Hellmann MD, Ciuleanu TE, Pluzanski A, et al. Nivolumab plus Ipilimumab in lung cancer with a high tumor mutational burden. $\mathrm{N}$ Engl J Med. 2018;378:2093-104.

44. Hagemann AR, Hagemann IS, Cadungog M, et al. Tissue-based immune monitoring II: multiple tumor sites reveal immunologic homogeneity in serous ovarian carcinoma. Cancer Biol Ther. 2011;12:367-77.

45. Wallace TJ, Qian J, Avital I, et al. Technical feasibility of tissue microarray (TMA) analysis of tumor-associated immune response in prostate cancer. J Cancer. 2018;9:2191-202.

46. Mahmoud SM, Paish EC, Powe DG, et al. Tumor-infiltrating CD8 + lymphocytes predict clinical outcome in breast cancer. J Clin Oncol. 2011;29:1949-55.

47. Erdag G, Schaefer JT, Smolkin ME, et al. Immunotype and immunohistologic characteristics of tumor-infiltrating immune cells are associated with clinical outcome in metastatic melanoma. Cancer Res. 2012;72:1070-80.

48. Han S, Zhang C, Li Q, et al. Tumour-infiltrating CD4(+) and CD8 $(+)$ lymphocytes as predictors of clinical outcome in glioma. Br J Cancer. 2014;110:2560-8.

49. Hardisson D, Moreno-Bueno G, Sanchez L, et al. Tissue microarray immunohistochemical expression analysis of mismatch repair (hMLH1 and hMSH2 genes) in endometrial carcinoma and atypical endometrial hyperplasia: relationship with microsatellite instability. Mod Pathol. 2003;16:1148-58.

50. Hendriks Y, Franken P, Dierssen JW, et al. Conventional and tissue microarray immunohistochemical expression analysis of mismatch repair in hereditary colorectal tumors. Am J Pathol. 2003;162:469-77

51. Visser NCM, van der Wurff AAM, Pijnenborg JMA, et al. Tissue microarray is suitable for scientific biomarkers studies in endometrial cancer. Virchows Arch. 2018;472:407-13. 